\title{
Semiconductor Nanorod Self-Assembly at the Liquid/Air Interface Studied by in Situ GISAXS and ex Situ TEM
}

\author{
Francesca Pietra, $^{\dagger}$ Freddy T. Rabouw, $^{\dagger}$ Wiel H. Evers, ${ }^{\dagger}$ Dima V. Byelov, ${ }^{\ddagger}$ Andrei V. Petukhov, ${ }^{\ddagger}$
} Celso de Mello Donegá, ${ }^{\dagger}$ and Daniël Vanmaekelbergh ${ }^{*}{ }^{\dagger}$

${ }^{\dagger}$ Condensed Matter and Interfaces, Debye Institute for Nanomaterials Science, Princetonplein 1, 3584 CC Utrecht, The Netherlands

${ }^{\ddagger}$ Physical and Colloid Chemistry, Debye Institute for Nanomaterials Science, Padualaan 8, $3584 \mathrm{CH}$ Utrecht, The Netherlands

\section{Supporting Information}

ABSTRACT: We study the self-assembly of colloidal CdSe/CdS nanorods (NRs) at the liquid/air interface combining timeresolved in situ grazing-incidence small angle X-ray scattering (GISAXS) and ex situ transmission electron microscopy (TEM). Our study shows that NR superstructure formation occurs at the liquid/air interface. Short NRs self-assemble into micrometers long tracks of NRs lying side by side flat on the surface. In contrast, longer NRs align vertically into ordered superstructures. Systematic variation of the NR length and initial concentration of
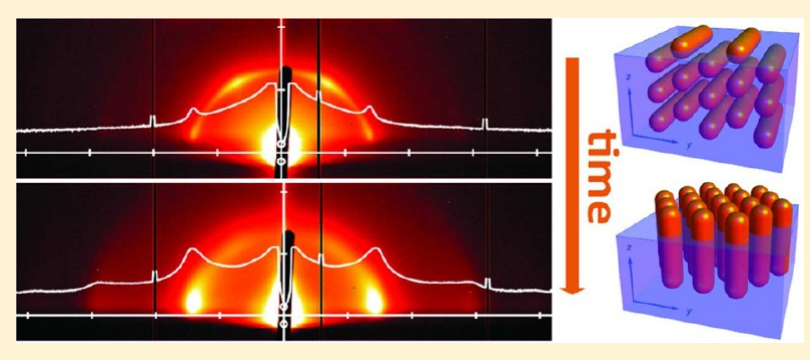
the NR dispersion allowed us to tune the orientation of the NRs in the final superstructure. With GISAXS, we were able to follow the dynamics of the self-assembly. We propose a model of hierarchical self-organization that provides a basis for the understanding of the length-dependent self-organization of NRs at the liquid/air interface. This opens the way to new materials based on NR membranes and anisotropic thin films.

KEYWORDS: Nanocrystals, self-assembly, superlattice, grazing-incidence small-angle X-ray scattering

$\mathrm{T}$ he ability to design the chemical and physical properties of colloidal nanocrystals (NCs) at the atomic level, combined with the possibilities of NC self-assembly is of great fundamental and applied interest. Self-assembly of NCs is a promising route toward the fabrication of new classes of materials in which collective properties may emerge as consequence of dipolar or quantum coupling between the NC building blocks. 1,2 NC superlattices and NC "membranes" are increasingly exploited as tailored nanostructured materials in optoelectronic devices such as LEDs, ${ }^{3-5}$ lasers $^{6}$ and solar cells. $^{7-10}$ Direct formation of nanocrystal superlattices at the liquid/air interface is currently emerging as a promising method to fabricate functional ultrathin films. Previous works reported promising results on the formation of $\left(\mathrm{mm}^{2}\right.$ large $)$ areas of thin films of binary and ternary superlattices composed of spherical NCs. These studies enhanced our understanding of the driving forces underlying NCs self-assembly. ${ }^{11-17}$

A new direction in this field is self-assembly of highly anisotropic nanocrystals, ${ }^{18}$ such as nanorods (NRs). ${ }^{19}$ The formation of vertically aligned and hexagonally packed superlattices was initially performed using an external electric field during the process of solvent evaporation. ${ }^{20,21}$ Subsequent efforts, however, have demonstrated that vertically aligned NR superlattices can be obtained by controlled solvent evaporation in the absence of any external forces. ${ }^{22-34}$ Self-assembly parameters like the aspect ratio of the NRs, evaporation temperature, and type of solvent were extensively explored. ${ }^{35}$ However, only the final structures were studied. To our knowledge, no in situ investigation of the dynamics of the selfassembly process has been reported, yet.

Here, we present a study of the mechanism of and the final structures formed by semiconductor NRs self-assembly. We observed that self-assembly occurs at the suspension/air interface and consists of several steps. The dynamics of the self-assembly process and the final structures formed depend critically on the NR length and on the concentration of the NR dispersion. We have used suspensions of $\mathrm{CdSe}(\mathrm{dot}) / \mathrm{CdS}$ NRs with narrow size and shape distribution and different aspect ratios, drop-casted on a layer of immiscible liquid that acts as a substrate. Self-assembly was initiated by evaporation of the solvent and large areas of organized structures were obtained. We studied the process of NRs self-assembly in situ by grazingincidence small angle X-ray scattering (GISAXS). ${ }^{36-41}$ The use of diethylene glycol (DEG) as substrate allowed us to keep the position of the liquid/air interface at constant height with respect to the X-ray beam during solvent evaporation.

For the chemical synthesis of $\mathrm{CdSe} / \mathrm{CdS}$ dot core/rod shell NRs, we adapted the method of Carbone et al. ${ }^{25}$ (see Supporting Information). This method allows for control over the length of the NRs by tuning the CdSe seed concentration and by adjusting temperature and reaction time. We determined the size of the NRs obtained from

Received: June 25, 2012

Revised: October 2, 2012

Published: October 5, 2012 
transmission electron microscopy (TEM) images. The NRs concentration was estimated using inductively coupled plasma atomic emission spectroscopy (ICP-AES). We used three different batches of NRs with narrow size distribution: (i) long NRs with length $L=(48 \pm 4) \mathrm{nm}$ and diameter $2 R=(4.1 \pm$ $0.4) \mathrm{nm}$, (ii) medium-length $\mathrm{NR}$ with $L=(22.2 \pm 2.3) \mathrm{nm}$ and $2 R=(6.3 \pm 1.0) \mathrm{nm}$, and (iii) short NRs with $L=(16.3 \pm 2.4)$ $\mathrm{nm}$ and $2 R=(3.4 \pm 0.3) \mathrm{nm}$ (Figure S1, Supporting Information). The NRs have octadecylphosphonic acid (ODPA) surface capping. We stored the $\mathrm{NRs}$ in $\mathrm{N}_{2}$ atmosphere.

Study of the Mechanism of NR Self-Assembly by in Situ GISAXS. In the first part of this study we used in situ GISAXS to follow the dynamics of NR self-assembly. The experiment was performed at the ID10B beamline of the European Synchrotron Radiation Facility (ESRF) with an incident X-ray energy of $13.3 \mathrm{keV}$. We used a grazing incidence angle of $\alpha_{i}=0.061^{\circ}$ for which there is total external reflection. In this way, we ensure that the beam only probes the toluene/ air interface at most $10-20 \mathrm{~nm}$ deep into the NRs dispersion (see Supporting Information for a experimental details and calculation of the penetration depth of the beam).

We carried out the self-assembly in a Teflon disk-shaped container with an inner volume of $11.3 \mathrm{~mL}$ ( $3.5 \mathrm{~mm}$ height; 64 $\mathrm{mm}$ base diameter). By using a container with high surface-tovolume ratio we ensured that (i) solvent evaporation happens over the course of $30 \mathrm{~min}$, (ii) the meniscus is flat in the region measured. A schematic drawing of the setup used is shown in Figure 1. For each of the three batches of NRs, the dispersion in toluene had an initial concentration of $1.6 \mu \mathrm{M}$. $4 \mathrm{~mL}$ of NR dispersion was deposited on top of $8 \mathrm{~mL}$ of DEG. The evaporation rate of toluene was set by heating the sample from the bottom with a copper stage at $T=50{ }^{\circ} \mathrm{C}$ and by gently flowing $\mathrm{N}_{2}$ over the sample. To keep the liquid level at constant height with respect to the incident $\mathrm{X}$-ray beam, we

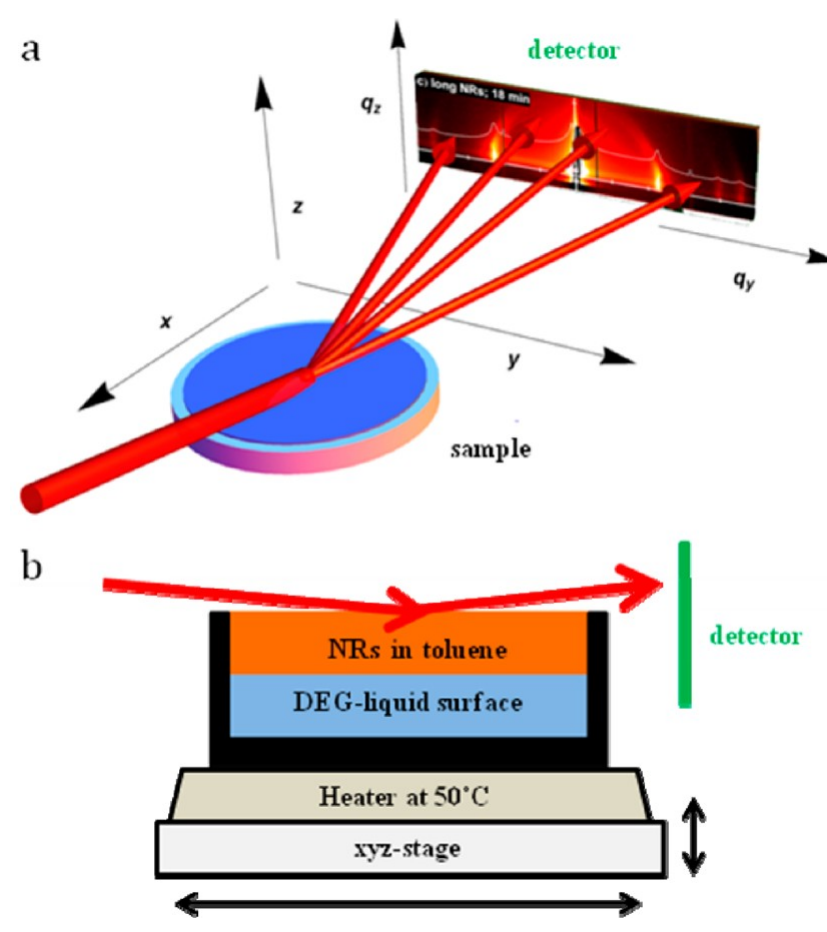

Figure 1. Representation of GISAXS experiment geometry (a) and cross sectional sketch of the setup (b). compensated for the evaporation of toluene by injecting additional DEG directly into the bottom liquid layer with an average addition rate of $0.13 \mathrm{~mL} / \mathrm{min}$. Every minute a scattering pattern was recorded by integrating over $30 \mathrm{~s}$.

Self-Assembly of Long NRs. Figure 2 shows three GISAXS patterns of the sample of the long NRs $(L=48$ $\mathrm{nm}$ ). The patterns were recorded at different stages of the selfassembly process: (a) $10 \mathrm{~min}$, (b) $14 \mathrm{~min}$, and (c) $18 \mathrm{~min}$ after the beginning of solvent evaporation. The whole sequence of patterns from 1 to $22 \mathrm{~min}$ can be found in Figure S3 (Supporting Information). The $10 \mathrm{~min}$ pattern (Figure 2a) shows a diffraction ring of radius $q_{1}=1.12 \mathrm{~nm}^{-1}$. We assign this ring to diffraction on $2 \mathrm{~d}$ hexagonal structures, as depicted in Figure $2 \mathrm{~d}^{42}$ The lattice distance $a=4 \pi / \sqrt{3} q_{1}=6.5 \mathrm{~nm}$ corresponds to NRs (diameter $2 R=4.1 \mathrm{~nm}$ ) lying side-to-side separated by two layers of ODPA ligands. The scattering intensity is constant along the diffraction ring, indicating that the structures are randomly oriented in the $y z$-plane. From this two-dimensional $\left(q_{y}-q_{z}\right)$ diffraction pattern we cannot distinguish whether the structures have totally random orientations in three dimensions, or the NRs are still preferentially oriented with their long axis parallel to the interface. In the $14 \mathrm{~min}$ pattern (Figure $2 \mathrm{~b}$ ), the diffraction ring of radius $q_{1}=1.12 \mathrm{~nm}^{-1}$ no longer has a constant intensity along the ring. There is considerably stronger diffraction toward $q_{z}=0$, indicating that the NRs now preferentially have an upstanding orientation. There are additional diffraction peaks near $q_{z}=0$ at $q_{y 2}=1.94 \mathrm{~nm}^{-1}$ and $q_{y 3}=2.24 \mathrm{~nm}^{-1}$. This sequence of $q_{y 1}: q_{y 2}: q_{y 3}=1: \sqrt{ } 3: 2$ near $q_{z}=0$ is characteristic for 2D hexagonal structures on the liquid/air interface, as depicted in Figure 2e. Furthermore, the diffraction ring of radius $q_{1}=$ $1.12 \mathrm{~nm}^{-1}$ consists of two rings with a $q_{z}$-offset corresponding to twice the angle of incidence of the X-ray probe beam. The two rings originate from diffraction of the nonreflected direct $\mathrm{X}$-ray beam (bottom ring) and the reflected X-ray beam (top ring). Diffraction of the reflected beam can only occur if structures are (partially) sticking out of the surface. This is an additional proof that the NRs form upstanding structures as depicted in Figure 2e. The $18 \mathrm{~min}$ pattern (Figure $2 \mathrm{c}$ ) is very similar to the $14 \mathrm{~min}$ pattern (Figure $2 \mathrm{~b}$ ), indicating that the vertically aligned NR superlattice structures have not changed meanwhile. The diffraction peaks of Figure $2 c$ have roughly the same width as in Figure $2 \mathrm{~b}$, but increased intensity. We conclude that between $14 \mathrm{~min}$ (Figure 2b) and $18 \mathrm{~min}$ (Figure 2c) after the beginning of solvent evaporation the area of liquid surface covered with the $2 \mathrm{D}$ hexagonal lattice (Figure 2e) has increased but the range of the hexagonal order has remained constant.

Self-Assembly of Medium-Length NRs. Figure 3 shows three GISAXS patterns of the sample of medium-length NRs ( $L$ $=22 \mathrm{~nm}$ ). The patterns were recorded at different stages of the self-assembly process: (a) 12 , (b) 14 , and (c) 16 min after the beginning of solvent evaporation. The whole sequence of patterns from 1 to $39 \mathrm{~min}$ can be found in Figure S4 (Supporting Information). The $12 \mathrm{~min}$ pattern (Figure 3a) shows a diffraction ring of radius $q_{1}=0.73 \mathrm{~nm}^{-1}$. We assign this ring to diffraction on a $2 \mathrm{D}$ hexagonal structure of NRs. The lattice distance $a=4 \pi / \sqrt{ } 3 q_{1}=9.9 \mathrm{~nm}$ corresponds to NRs (diameter $2 R=6.3 \mathrm{~nm}$ ) lying side-to-side separated by two layers of ODPA ligands. We note that the apparent spacing between the medium-length NRs $(3.6 \mathrm{~nm})$ is somewhat larger than expected considering the length of the ligands. This possibly can be explained by considering that the medium- 

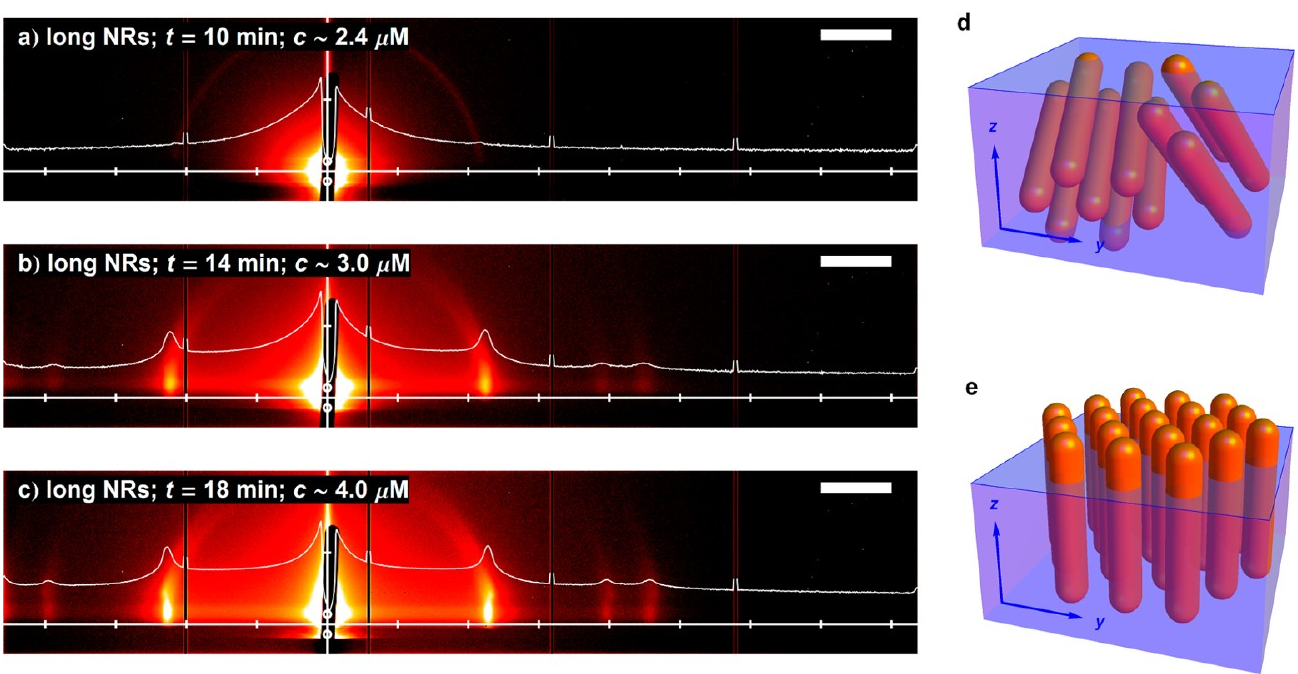

Figure 2. GISAXS patterns of the superstructures of long NRs $(L=48 \mathrm{~nm})$ at the liquid/air interface at different stages after the beginning of solvent evaporation: (a) 10, (b) 14, and (c) $18 \mathrm{~min}$. Color scales are logarithmic. Scale bars are $0.5 \mathrm{~nm}^{-1}$. For each pattern the estimated NR concentration $c$ is shown. $(\mathrm{d}, \mathrm{e})$ Schematics of the NRs structures as present $(\mathrm{d})$ at the beginning [pattern (a)], and (e) at the end [patterns (b,c)] of the selfassembly process.
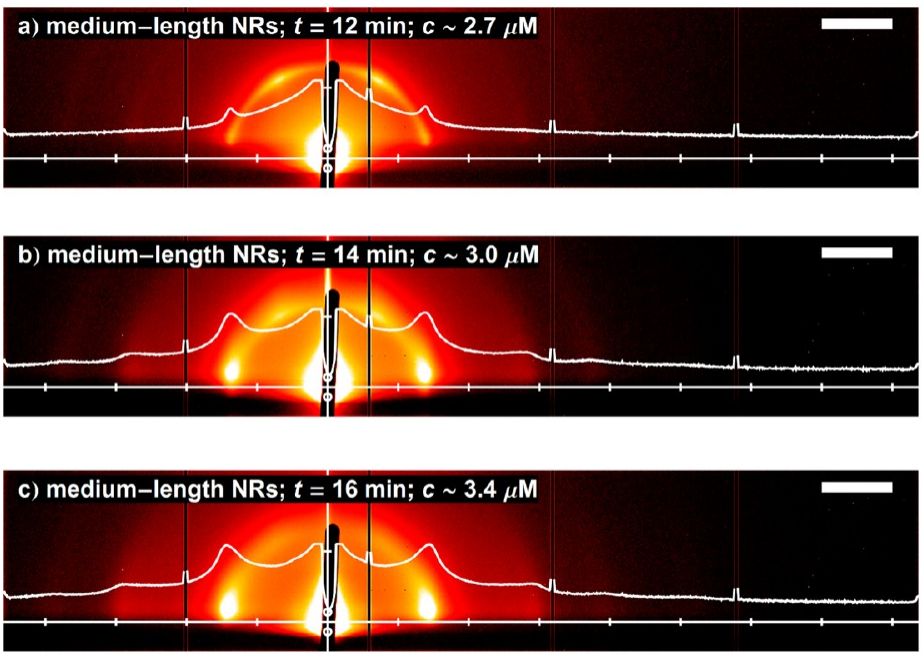

d
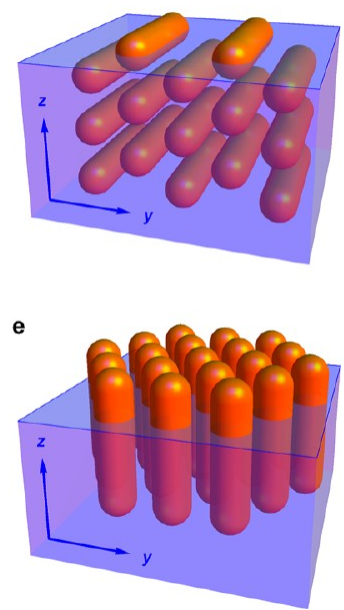

Figure 3. GISAXS patterns of the superstructures of medium-length NRs $(L=22 \mathrm{~nm})$ at the liquid/air interface at different stages after the beginning of solvent evaporation: (a) 12, (b) 14, and (c) 16 min. Color scales are logarithmic. Scale bars are $0.5 \mathrm{~nm}^{-1}$. For each pattern the estimated NR concentration $c$ is shown. (d,e) Schematics of the NRs structures as present (d) at the beginning [pattern (a)], and (e) at the end [pattern (c)] of the self-assembly process.

length NRs have a large diameter polydispersity: $\sigma_{2 \mathrm{R}}=1.0 \mathrm{~nm}$; hence, the superstructure of medium-length NRs has to be slightly more expanded in order to also accommodate the thicker NRs of the ensemble. This results in a larger average lattice constant and thus an increased average inter-NR distance. $^{44}$ Along the diffraction ring (Figure 3a) there are four spots of increased intensity at $\theta_{q}=\{0, \pi / 3,2 \pi / 3, \pi\}$. These spots indicate that the preferential orientation of the hexagonal lattice is with a zigzag edge adsorbed to the liquid/air interface, as depicted in Figure 3d. We now look at the $14 \mathrm{~min}$ pattern (Figure 3b). The two scattering peaks at $\theta_{q}=\{\pi / 3,2 \pi /$ $3\}$ have become broader than in Figure $3 a$, and the two peaks near $q_{z}=0\left(\theta_{q}=\{0, \pi\}\right)$ have increased intensity (see also Figure S12 i Supporting Information for the azimuthal angle distribution of scattering intensity along the ring). Furthermore, we now clearly observe the higher order diffraction rings of radii $q_{2}=1.25 \mathrm{~nm}^{-1}, q_{3}=1.45 \mathrm{~nm}^{-1}, q_{4}=1.9 \mathrm{~nm}^{-1}$. The ratio of $q_{1}: q_{2}: q_{3}: q_{4}=1: \sqrt{ } 3: 2: \sqrt{ } 7$ is characteristic for hexagonal structures. We argue that this pattern is intermediate between the initial structure [12 min; Figure 3a,d] and the final structure [16 min; Figure 3c,e]. In the $16 \mathrm{~min}$ pattern (Figure 3c) the two scattering peaks at $\theta_{q}=\{\pi / 3,2 \pi / 3\}$ have almost disappeared. The two peaks at $q_{z}=0\left(\theta_{q}=\{0, \pi\}\right)$, on the other hand, have become even stronger than in Figure $3 \mathrm{~b}$. Higher order diffractions are still visible. In-plane scattering (near $q_{z}=0$ ) dominates over out-of-plane scattering, indicating that there is strong hexagonal ordering in the plane parallel to the toluene/air interface. We assign the pattern to diffraction on a $2 \mathrm{D}$ hexagonal lattice of upstanding NRs at the toluene/air interface as in Figure 3e. Upon going from Figure 3a to Figure $3 \mathrm{c}$ we have seen that at first the medium-length NRs lie down with their long axis parallel to the liquid/air interface (Figure $3 \mathrm{~d}$ ), while over the course of $4 \mathrm{~min}$ the NRs rise up into a 

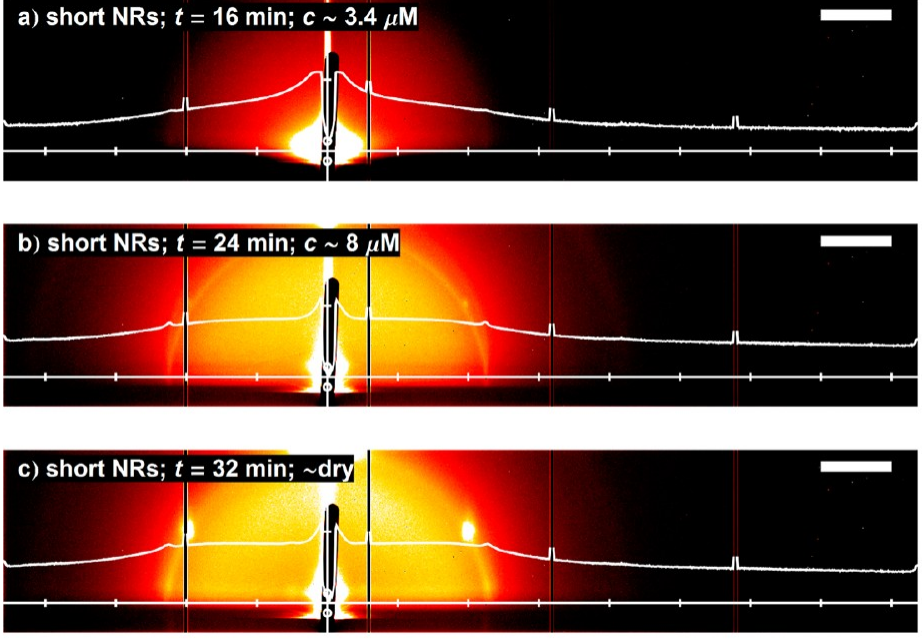

Figure 4. GISAXS patterns of superstructures of short NRs $(L=16 \mathrm{~nm})$ at the liquid/air interface at different stages of the solvent evaporation: (a) 16, (b) 24, and (c) $32 \mathrm{~min}$. Color scales are logarithmic. Scale bars are $0.5 \mathrm{~nm}^{-1}$. For each pattern the estimated NR concentration $c$ is shown. (d,e) Schematics of the NRs structures as present (d) at the beginning [pattern (b)], and (e) at the end [pattern (c)] of the self-assembly process.

vertical position forming an upstanding superstructure (Figure 3e).

Self-Assembly of Short NRs. Figure 4 shows three GISAXS patterns of the sample of short NRs $(L=16 \mathrm{~nm})$. The patterns were recorded at different stages of the selfassembly process: (a) 16, (b) 24, and (c) $32 \mathrm{~min}$ after the beginning of solvent evaporation. The whole sequence of patterns from 1 to $32 \mathrm{~min}$ can be found in Figure S5 (Supporting Information). The $16 \mathrm{~min}$ pattern (Figure 4a) shows no order yet. The background scattering is dominated by the form factor and no clear structure factor features are observed. The 24 min pattern (Figure $4 \mathrm{~b}$ ) shows a diffraction ring of radius $q_{1}=1.15 \mathrm{~nm}^{-1}$. We assign this ring to diffraction on $2 \mathrm{D}$ hexagonal structures as depicted in Figure $4 \mathrm{~d}$. The lattice constant $a=4 \pi / \sqrt{ } 3 q_{1}=6.3 \mathrm{~nm}$ corresponds to NRs (diameter $2 R=3.4 \mathrm{~nm}$ ) lying side-to-side separated by two layers of ODPA ligands. From the fact that the intensity is constant over the whole ring, we conclude that the hexagonal structures have random orientations in the $y z$-plane. From this two-dimensional $\left(q_{y}-q_{z}\right)$ diffraction pattern, we cannot distinguish whether the structures have totally random orientations in three dimensions, or the NRs are still preferentially oriented with their long axis parallel to the interface.

Comparing this pattern (Figure $4 \mathrm{~b}$ ) to those of the other samples (Figures 2 and 3 ) we see that the background scattering is markedly higher here. This might be indicative of a higher concentration of single particles and smaller bundles of NRs. In the $32 \mathrm{~min}$ pattern (Figure 4c), we still see the diffraction ring of radius $q_{1}=1.15 \mathrm{~nm}^{-1}$. There are now three spots of increased intensity on the ring at $\theta_{q}=\{\pi / 6,3 \pi / 6,5 \pi /$ $6\}$. These three spots indicate that the hexagonal structures now have a preferred orientation with the close-packed facets of the NR supertructure adsorbed to the toluene/air interface as illustrated in Figure 4e. In addition, we see two tiny peaks at $q_{0}$ $=0.25 \mathrm{~nm}^{-1}$ corresponding to scattering on planes separated by $d_{0}=25 \mathrm{~nm}$. This distance is close to what we would expect for head-to-tail packing of these NRs (length $L=16.5 \mathrm{~nm}$ plus two layers of ODPA ligands) (see also Supporting Information for a basic explanation of the origin of the diffraction ring peaks for short NRs). From these two peaks, we conclude that there is not only hexagonal side-to-side ordering of lying NRs (Figure 4e), but that the so-formed NR tracks also align head-to-tail. This conclusion is corroborated by the TEM results presented below. We note that the lying final structure formed by these short NRs and the lying intermediate structure of the mediumlength NRs (Figure $3 \mathrm{~b}$ ) have a different orientation by $60^{\circ}$ with respect to the liquid/air interface. However, with the present limited data set we are unable to give an explanation for this remarkable observation. This result does not affect our interpretation of the length dependent self-assembly, the main issue of the presented work.

We emphasize that even in the latest frames depicted for the long (Figure 2c) and the medium-length (Figure 3c) NR batches still only part of the initial toluene volume had evaporated. At these stages, the bulk NR dispersions were still rather dilute (NR volume fraction $<10^{-2}$ ). Yet, colloidal crystallization has started as strong ordering is observed at the liquid/air interface. On the other hand, ror the short NRs colloidal crystallization at the liquid/air interface does not take place until after $24 \mathrm{~min}$ (Figure $4 \mathrm{~b}$ ). At this stage, the NR suspension is already almost dry. Clearly, short NRs require a much higher concentration before colloidal crystallization sets in. As can be seen in the full sequence of GISAXS patterns (Figures S2, S3, and S4 in Supporting Information), the latetime patterns for the long (Figure 2c) and medium-length NRs (Figure 3c) during solvent evaporation are very similar to the final structures observed after complete drying. This shows that the top layer of the long and medium-length NR superstructures is fully formed as early as after $50 \%$ of the toluene solvent has been evaporated. For each of the three NR lengths, Figure S5, S6 and S7 in Supporting Information show six patterns recorded at different positions along the sample surface $(y=-6,-4,-2,2$, and $4 \mathrm{~mm}$ from the center of the sample) after complete solvent evaporation. For each NR length, the six patterns are very similar, indicating that the structures we observed in our time-resolved GISAXS study are representative for the entire sample surface. We can conclude that the $2 \mathrm{~d}$ hexagonal lattice is preserved during solvent evaporation and final drying, and it is present in large areas. Note that for all scattering patterns recorded, the scattering can only originate from structures residing close to the liquid/air interface, since the X-ray beam probes no further than $10-20$ 


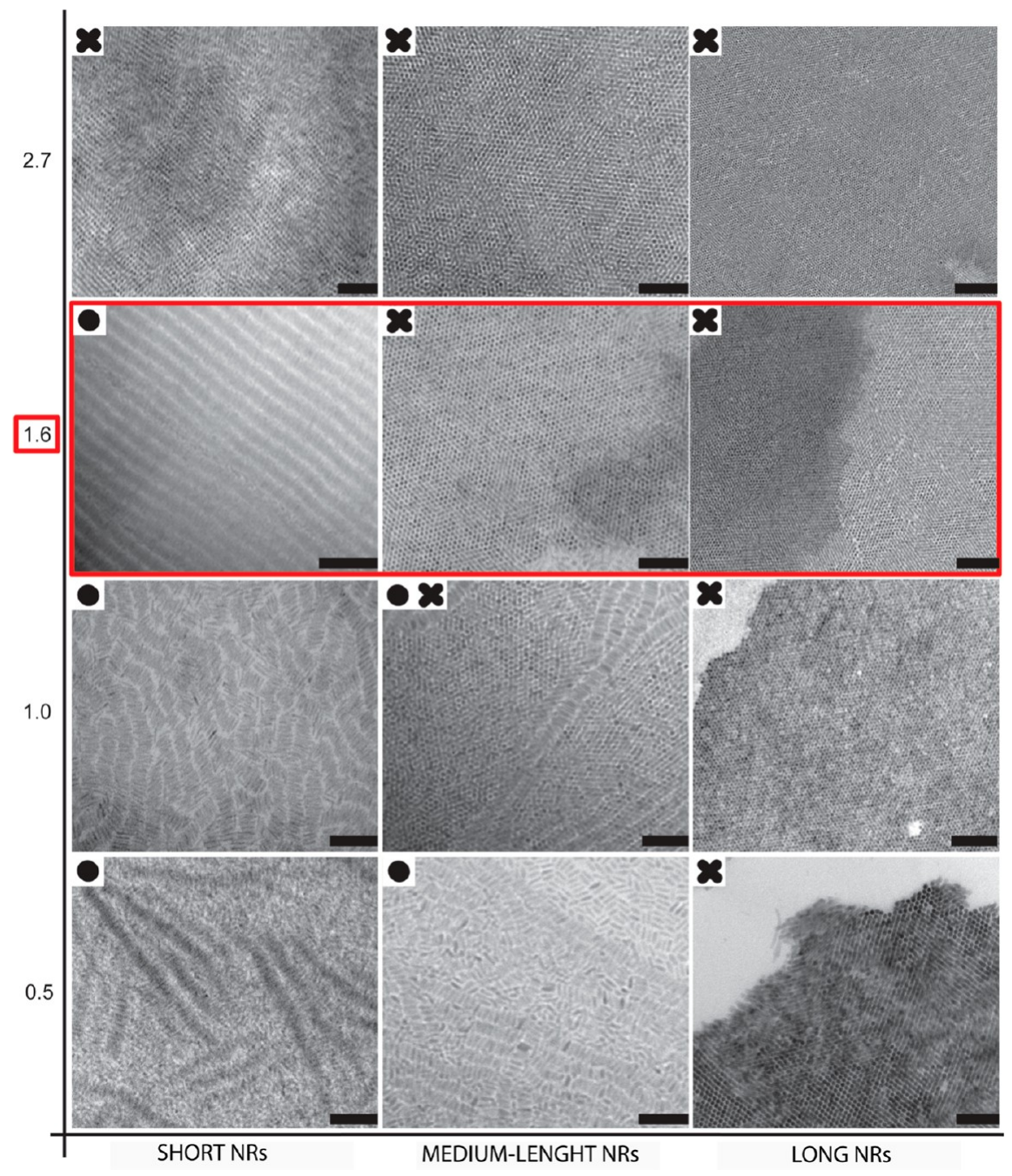

Figure 5. Overview of the final structures self-assembled from NRs of varying length ( $x$-axis) and from NR dispersions of varying initial concentrations ( $y$-axis). Scale bars are $50 \mathrm{~nm}$. Hexagonally ordered upstanding structures are marked with a cross in the upper left corner; NRs lying flat and side-by-side are marked with a circle. For the GISAXS experiments, we used an initial NR concentration of $1.6 \mu \mathrm{M}$, corresponding to the second row of the overview (highlighted).

$\mathrm{nm}$ deep into the toluene layer. Therefore, for upstanding NRs we only see diffraction on the upper half of the NRs layer, since the lengths of the NRs exceeds the penetration depth of the Xray beam. For NRs lying down we probe a few layers of NRs below the top one, since the NR diameter is of the order of the penetration depth. We also remark that all structure factor features (except one) are centered around the point $\left(q_{y}, q_{z}\right)=$ $\left(0,-q_{i}\right)$, that is, the point where the direct nonreflected beam would hit the detector. Only the top ring observed in the latetime patterns of long NRs (Figure $2 \mathrm{~b}, \mathrm{c})$ is centered around $\left(q_{y}\right.$, $\left.q_{z}\right)=\left(0,+q_{i}\right)$, that is, the point where the reflected beam hits the detector. We conclude that all structures are fully immersed under the toluene/air interface, except the upstanding structures of long NRs, which are partly sticking out. If, and only if, a structure is (partly) sticking out of the liquid, it can diffract the X-ray beam either before or after reflection from the surface.

With this GISAXS study, we have demonstrated that at a fixed concentration the NRs self-assemble into different structures depending on their length, and that NR ordering into superstructures takes place at the toluene/air interface. For the long NRs, we only find evidence for a hexagonally ordered upstanding structure at the toluene/air interface (Figure 2e). For the medium-length NRs, we were able to follow the transition from an intermediate state (Figure 3d) in which the NRs are hexagonally ordered lying down at the liquid/air interface to the final superstructure (Figure 3e) consisting of hexagonally ordered upstanding NRs. For the short NRs, we only observed a hexagonally ordered structure with the NRs lying down (Figure 4e) that only forms at late stages of the selfassembly process.

TEM Study of the NR Superlattices. In a second set of experiments, we aimed to investigate the final structure of the NR superlattices that are formed upon solvent evaporation for NRs with different lengths and using NR suspensions with different initial concentrations. To this end we used ex situ TEM. In the experiments, we systematically varied the NR concentration in toluene between 0.5 and $2.7 \mu \mathrm{M}$. We loaded $50 \mu \mathrm{L}$ of NR dispersion in a $5.3 \mathrm{~mL}(3.5 \mathrm{~cm}$ height; $1.4 \mathrm{~cm}$ inner diameter) Teflon container on top of $4.5 \mathrm{~mL}$ of an immiscible DEG liquid layer. The sample was heated from the bottom to $T=50{ }^{\circ} \mathrm{C}$. After $30 \mathrm{~min}$, the evaporation of the toluene was complete. Then, a sample of the self-assembled structure was directly fished with a copper TEM grid from a 

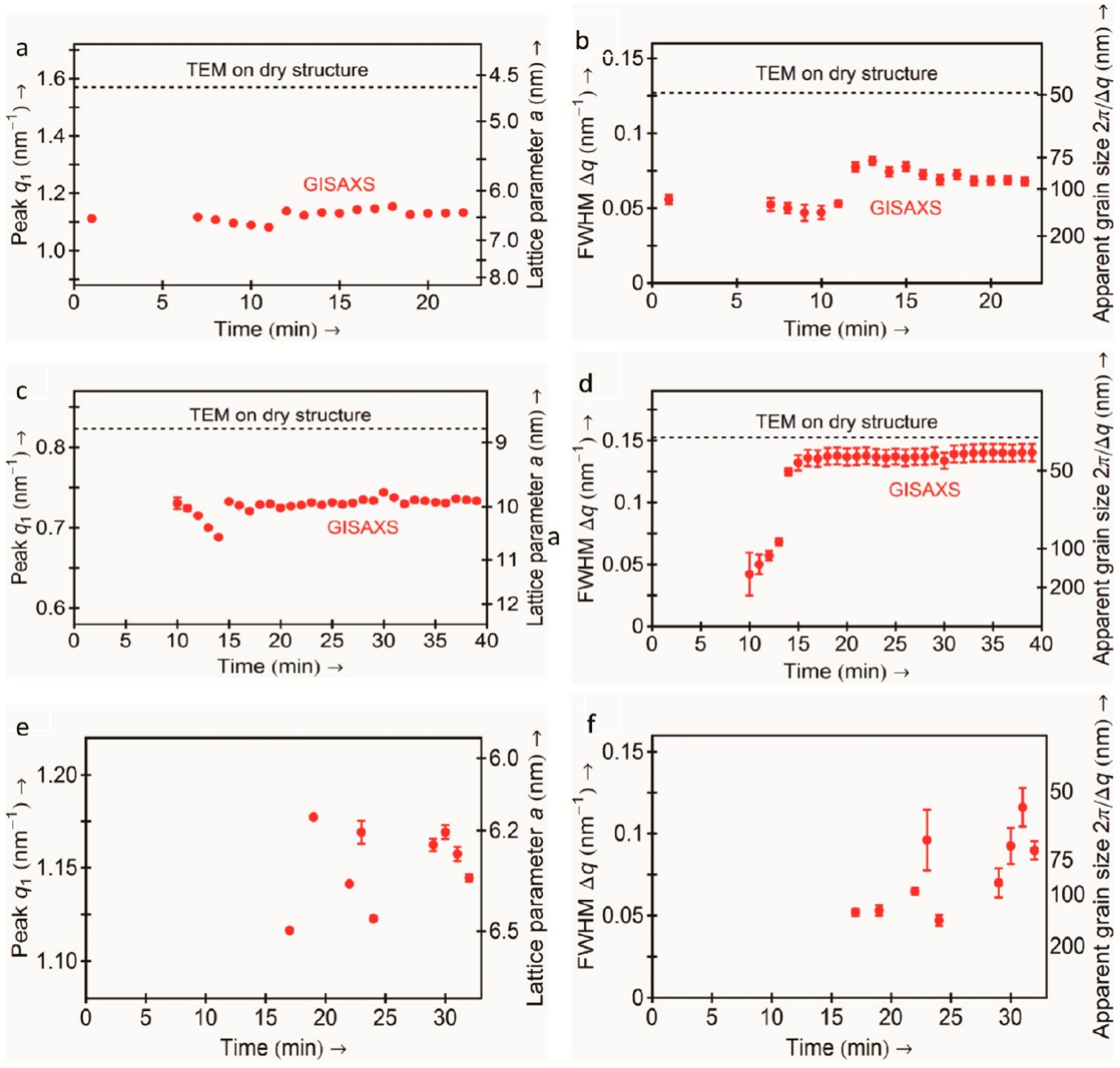

Figure 6. Fit of the GISAXS patterns and the FFT-TEM images for the three samples analized. $(\mathrm{a}, \mathrm{b})$ Long NRs; (c,d) medium-length NRs; and (e,f) short NRs. The graphs show plots of the peak position $q_{1}(\mathrm{a}, \mathrm{c}, \mathrm{e})$ (lattice parameter $a$ ) and full-width-at-half-maximum (fwhm) $\Delta q$ (apparent grain size $2 \pi / \Delta q)(\mathrm{b}, \mathrm{d}, \mathrm{f})$ of the first structure factor peak for the in situ samples as a function of time (dots). Dashed lines indicate the peak position and fwhm of the first structure factor peak of the fully dried samples.

random position on the DEG/air interface. TEM images were made in a Tecnai microscope operating at $120 \mathrm{kV}$.

For each of the three NR lengths, we performed the selfassembly using four different initial NR concentrations: $0.5,1$, 1.6 , and $2.7 \mu \mathrm{M}$. Figure 5 shows a complete overview of the twelve self-assembled structures formed. Note that we carried out all experiments in duplicate and obtained similar results the second time. TEM images of hexagonally ordered upstanding structures are marked with a cross in the upper left corner. TEM images of structures with the NRs lying flat side-by-side are marked with a circle. There is one sample that shows a mixture of upstanding and lying structures. The lying structures (circles) show long tracks of NRs packed side-by-side. From the GISAXS experiments, we learned that the tracks of NRs extend into the bulk suspension (i.e., they are not monolayers) with a hexagonal ordering within the tracks. The overview shows that the NRs tend to organize into lying structures at low initial concentration evolving into upstanding structures at higher initial NR concentrations. The transition from lying-toupstanding occurs at lower initial concentration for longer NRs. For the lying structures in the overview (circles in Figure 5), we see that very low concentrations lead to a partial organization with positional order but no orientational order. With increasing the concentration, the tracks become longer and the mutual alignment increases. Our TEM observations are fully in line with what we found from the GISAXS experiments.
For the GISAXS experiments, we used an initial concentration of $1.6 \mu \mathrm{M}$, corresponding to the second row in Figure 5. From GISAXS we found that long and medium-length NRs eventually form an upstanding structure, whereas short NRs end up lying flat on the toluene/air surface. The same trend is visible in the second row of Figure 5.

Analysis of the Temporal Evolution of the SelfAssembled Superstructures. For all the GISAXS patterns recorded and for some fast Fourier transform TEM (FFTTEM) (second row Figure 5), we determined the position and width of the first structure factor peak. This yields a quantitative analysis of the in situ temporal evolution of the structures, and a comparison between in situ structures measured with GISAXS and ex situ structures measured with TEM (see the Supporting Information for a more detailed explanation of the fitting procedure. Figure 6 shows the peak position $q_{1}$ (left column) and full-width-at-half-maximum (fwhm; right column) $\Delta q$ of the first structure factor peak for all three NR lengths for the in situ samples as a function of time, as determined from fits to the GISAXS patterns. Dashed lines indicate the peak position and fwhm of the first structure factor peak of the fully dried samples of medium and long NRs, as determined from fits to the FFT-TEM images. (Since from lying structures it is not possible to extract the ordering in the direction perpendicular to the long axis of the rods, we only analyzed FFT-TEM images for the long and medium-length NRs). From the peak position 

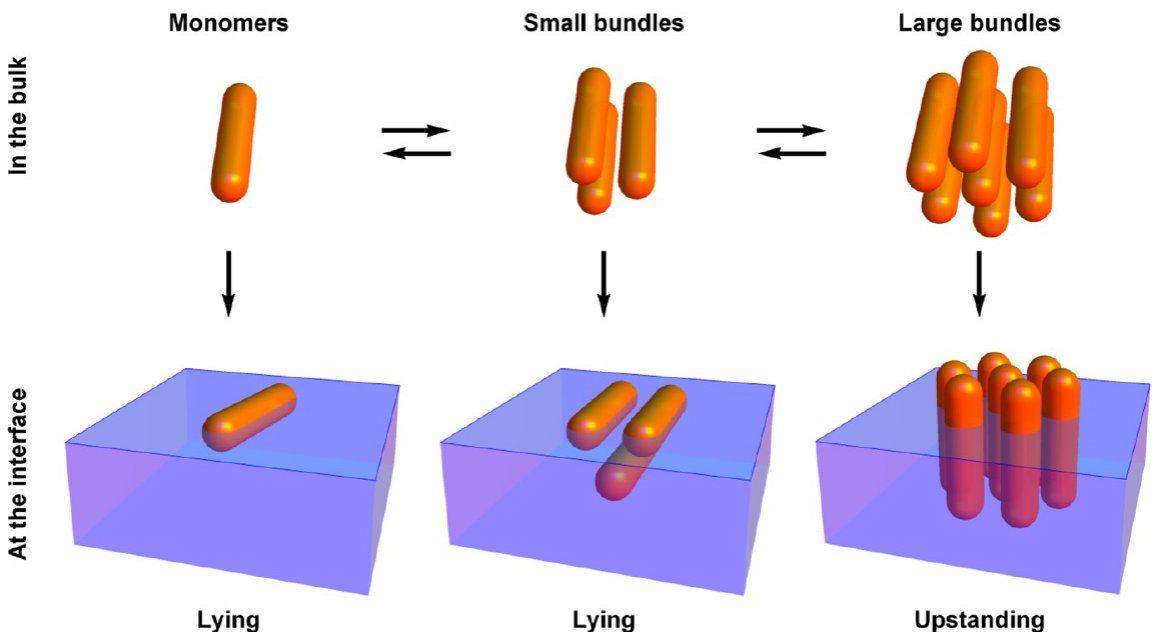

Figure 7. Schematic representation of step (i) and (ii) in our self-assembly model. In the bulk liquid single NRs are in equilibrium with NRs packed together into bundles. Single NRs or small bundles adsorb to the liquid/air interface in a lying orientation. Large bundles adsorb to the interface in an upstanding orientation.

and fwhm we calculate the lattice parameter $a=4 \pi / \sqrt{ } 3 q_{1}$ and the apparent grain size $D=2 \pi / \Delta q$ of the $2 \mathrm{D}$ hexagonal structure. The left column in Figure 6 shows that the peak positions $q_{1}$, and hence lattice parameters $a$, of the in situ structures (red dots) remain approximately constant over time (within the spread of the data points). We do not observe any shrinking of the superstructure as the toluene evaporates. For the fully dried superstructures (dashed lines), however, $q_{1}(a)$ is significantly larger (smaller) than for the corresponding in situ structure. For the long NRs, drying in vacuum $\left(10^{-3} \mathrm{mbar}\right)$ induces a shrinking of the superstructure from $a=6.5 \mathrm{~nm}$ to $a$ $=4.6 \mathrm{~nm}$. For the medium-length NRs, the superstructure shrinks from $a=9.9 \mathrm{~nm}$ to $a=8.8 \mathrm{~nm}$. These results show that during solvent evaporation the hexagonal array of the vertical rods is preserved and that the only further effect of extensive drying is a reduction of the distance between the rods, possibly due to interpenetration of the ligands.

The right column in Figure 6 shows that the peak widths $\Delta q$ for the long NR sample, the short NR sample, and for the late stages of the medium-length NR sample remain roughly constant over time. The only clear feature observed in the graphs is the transition from $\Delta q=0.05 \mathrm{~nm}^{-1}$ to $\Delta q=0.13$ $\mathrm{nm}^{-1}$ for the medium-length NRs around $t=15 \mathrm{~min}$ (see below). We explain the mostly constant peak width $\Delta q$ by arguing that $\Delta q$ is not determined by grain size, which we expect to grow as the NR superstructure becomes more crystalline. Instead, it may be determined by the presence of defects and dislocations that disturb the periodic order and induce a variation $\Delta a$ in the lattice constant. For our samples the peak broadening due to the distribution in the lattice constant $\Delta a$ is larger than the peak broadening due to the finite grain size. Hence, the apparent grain sizes calculated as $2 \pi / \Delta q$ in Figure 6 present the lower limits to the actual grain sizes. The lower limits to the actual grain sizes correspond to grains of approximately 15 unit cells for the long and short NRs, and 5 unit cells for the medium-length NRs. Unfortunately, we do not observe diffraction from smaller grains (i.e., superstructure nuclei) at early times. The absence of diffraction peaks from small grains is expected considering that the diffraction intensity scales with the square of the volume of the diffracting units. The smallest grains hardly diffract at all.
Given our explanation of the peak width, one expects that the variation $\Delta a$ in the lattice parameter, and hence peak width $\Delta q$, increases with increasing diameter polydispersity $\sigma_{2 R}$ of the NRs. Indeed, we observe a correlation between $\Delta q$ and $\sigma_{2 R}: \Delta q$ $=0.068 \mathrm{~nm}^{-1}, 0.124 \mathrm{~nm}^{-1}$, and $0.064 \mathrm{~nm}^{-1}$ (weighted average of the data points in Figure 6) and $\sigma_{2 R}=0.4,1.0$, and $0.3 \mathrm{~nm}$ for the long, medium-length, and short NRs respectively. The medium-length NRs (Figure 6d) are the only sample that do show a temporal evolution of $\Delta q$ : a transition from $\Delta q=0.05$ $\mathrm{nm}^{-1}$ to $\Delta q=0.13 \mathrm{~nm}^{-1}$ around $t=15 \mathrm{~min}$. This change in peak width coincides with the transition from a lying to an upstanding structure (see Figure 3). We propose the following tentative interpretation: the different values of $\Delta q$ for the lying and the upstanding structures support a seeded-growth type mechanism for the transition: an entirely new superstructure with a different variation $\Delta a$ in the lattice parameter, forms as NRs detach from the lying structure and attach to the upstanding structure. For grain rotation, another possible mechanism, one would expect $\Delta q$ to remain constant. The superstructures before and after grain rotation would have the same value of $\Delta a$, since they do not rearrange but only rotate. We should remark here that a seeded-growth type mechanism (however leading to a linear array of lying rods) has been proposed before. $^{19}$

Discussion. Here we present a mechanism of hierarchical self-assembly of NRs at the toluene/air interface. We extended the model proposed by Denkov et al. ${ }^{43}$ to our case of anisotropic particles, so that it can explain the NR length dependence and the initial NR concentration dependence of the superstructures we observe. The mechanism we propose for the self-assembly consists of three steps: (i) under the influence of mutual van der Waals (vdW), and possibly dipolar interactions the NRs form bundles in solution; (ii) adsorption of these bundles to the toluene/air interface lowers interfacial tension; (iii) the bundles pack together at the interface in such a way to maximize attractive (vdW) interactions. We can distinguish two possible scenarios. In scenario (a), the NRs remain as monomers or cluster into very small bundles of 2 or 3 NRs [step (i)]. These structures adsorb with the long NR axis parallel to the liquid/air interface [step (ii)]. The structures adsorbed pack together [step (iii)] to form the lying structures as observed on the bottom left of Figure 7 (short NRs and/or 
low NR concentration). In scenario (b), the NRs cluster into larger bundles of, for example, six or more NRs [step (i)]. A cross-section of these structures perpendicular to the long NR axis has approximately the same or an even larger area than a cross-section parallel to the long NR axis. Therefore, these structures may adsorb to the liquid/air interface in an upstanding orientation [step (ii)]. The bundles adsorbed pack together or act as nuclei onto which additional NRs can attach in an upstanding orientation [step (iii)], resulting in upstanding structures as observed on the top right of Figure 7 (long NRs and/or high NR concentration). Steps (i) and (ii) of the model are schematically depicted in Figure 7. The orientation of NRs in the final structure depends on the size of NRs bundles formed in the bulk dispersion, which in turn depends on the NRs concentration and length. The size of NR bundles increases if the bulk NRs concentration increases, or if the mutual NR-NR interactions become stronger. VdW interactions scale with the NR length. We performed some simple thermodynamic calculations of which the results support our model. They can be found in the Supporting Information. We calculate for the experimental conditions for which we find upstanding structures, that in the bulk a considerable fraction of NRs aggregate into (large) bundles. For the experimental conditions for which we find lying structures, the calculations show that the bundles are considerably smaller. The evolution from a superstructure with lying NRs (Figure 2a) into a structure with upstanding NRs (Figure 2c) as monitored by time-resolved GISAXS on the medium-length NRs can also be understood in terms of our model. At early stages of the selfassembly process, the concentration of NRs is low and only small bundles are present that adsorb in a lying orientation. As solvent evaporation continues, the NR concentration increases, and larger bundles form that adsorb in an upstanding orientation. We have observed that initially lying NRs rise up into an upstanding orientation. We can argue that as soon as a small fraction of adsorbed NRs have an upstanding orientation, these can act as nuclei onto which the upstanding superstructure grows if additional NRs attach.

We have studied the formation of large areas of vertically or horizontally aligned nanorods (NRs) at the liquid/air interface, obtained by controlled evaporation of the solvent using a liquid diethylene glycol substrate. We have proven that NR superstructure formation occurs at the liquid/air interface. In addition, we were able to partly follow the dynamics of selforganization. We have shown that the orientation of NRs in the final superstructure can be tuned by changing the NR length and the initial concentration in the NR dispersion. Using short NRs or a low concentration, structures form with NRs lying flat on the liquid/air interface. Using long NRs or a high concentration, structures form with NRs standing up. With NRs of medium length we been able to monitor in real time the dynamical process of NRs that rise up from a lying orientation into an upstanding orientation. We have proposed a model of hierarchical self-organization that accounts for the NR length and concentration dependence of the superstructures formed. Our results provide insight into the mechanism of the NR selfassembly at the liquid/air interface, opening the way for the exploration of new promising applications and new strategies for device engineering.

\section{ASSOCIATED CONTENT}

\section{Supporting Information}

Additional information and figures. This material is available free of charge via the Internet at http://pubs.acs.org.

\section{AUTHOR INFORMATION}

\section{Corresponding Author}

*E-mail: d.vanmaekelbergh@uu.nl. Tel.: +31 30-2532218. Fax: +31 30-2532403.

\section{Notes}

The authors declare no competing financial interest.

\section{ACKNOWLEDGMENTS}

The authors would like to thank Oleg Konovalov, Marjolein Dijkstra, and Jos van Rijssel for helpful discussion and critical review of our manuscript. EU Seventh Framework Program (EU-FP7 ITN Herodot, NMP-2009-1.2.-1 NanoSpec) and "Stichting voor Fundamenteel Onderzoek der Materie (FOM)", which is financially supported by the "Nederlandse Organisatie voor Wetenschappelijk Onderzoek (NWO)" (project 67595) are acknowledged for the financial support. Part of this work was carried out at the European Synchrotron Radiation Facility (ESRF) in Grenoble (France), experiment SC-3300.

\section{REFERENCES}

(1) Vanmaekelbergh, D. Nano Today 2011, 6, 419-437.

(2) Grzelczak, M.; Vermant, J.; Furst, E. M.; Liz-Marzán, L. M. ACS Nano 2010, 4, 3591-3605.

(3) Sun, B.; Sirringhaus, H. Nano Lett. 2005, 5, 2408-2413.

(4) Hu, J.; Li, L.,S.; Yang, W.; Manna, L.; Wang, L., W.; Alivisatos, A. P. Science 2001, 292, 2060-2063.

(5) Rizzo, A.; Nobile, C.; Mazzeo, M.; Giorgi, M. D.; Fiore, A.; Carbone, L.; Cingolani, R.; Manna, L.; Gigli, G. ACS Nano 2009, 3, $1506-1512$.

(6) Pisanello, F.; Martiradonna, L.; Spinicelli, P.; Fiore, A.; Hermier, J. P.; Manna, L.; Cingolani, R.; Giacobino, E.; De Vittorio, M.; Bramati, A. Superlattices Microstruct. 2010, 47, 165-169.

(7) Gonzalez-Valls, I.; Lira-Cantu, M. Energy Environ. Sci. 2009, 2, 19-34.

(8) Talgorn, E.; Gao, Y.; Aerts, M.; Kunneman, L. T.; Schins, J. M.; Sevenije, T. J.; van Huise, M. A.; van der Zant, H. S. J.; Houtepen, A. J.; Siebbeles, A. Nat. Nanotechnol. 2011, 6, 733-739.

(9) Huynh, W. U.; Dittmer, J. J.; Alivisatos, A. P. Science 2002, 295, 2425-2427.

(10) Tang, J.; Kemp, K. W.; Hoogland, S.; Jeong, K. S.; Liu, H.; Levina, L.; Furukawa, M.; Wang, X.; Debnath, R.; Cha, D.; Chou, K. W.; Fischer, A.; Amassian, A.; Asbury, J. B.; Sargent, E. H. Nat. Mater. 2011, 10, 765-771.

(11) Dong, A.; Chen, J.; Vora, P. M.; Kikkawa, J. M.; Murray, C. B. Nature 2010, 466, 474-477.

(12) Evers, W. H.; Nijs, B. D.; Filion, L.; Castillo, S.; Dijkstra, M.; Vanmaekelbergh, D. Nano Lett. 2010, 10, 4235-4241.

(13) Shevchenko, E. V.; Talapin, D. V.; Kotov, N. A.; O’Brien, S.; Murray, C. B. Nature 2006, 439, 55-59.

(14) Shevchenko, E. V.; Kortright, J.; Talapin, D. V.; Aloni, S.; Alivisatos, A. P. Adv. Mater. 2007, 19, 4183-4188.

(15) Talapin, D. V.; Shevchenko, E. V.; Bodnarchuk, M. I.; Ye, X.; Chen, J.; Murray, C. B. Nature 2009, 461, 964-967.

(16) Talapin, D. V.; Shevchenko, E. V.; Kornowski, A.; Gaponik, N.; Haase, M.; Rogach, A. L.; Weller, H. Adv. Mater. 2001, 13, 18681871.

(17) Talapin, D. V.; Shevchenko, E. V.; Murray, C. B.; Titov, A. V.; Král, P. Nano Lett. 2007, 7, 1213-1219. 
(18) Miszta, K.; de Graaf, J.; Bertoni, G.; Dorfs, D.; Brescia, R.; Marras, S.; Ceseracciu, L.; Cingolani, R.; van Roij, R.; Dijkstra, M.; Manna, L. Nat. Mater. 2011, 10, 872-876.

(19) Li, L. S.; Alivisatos, A. P. Adv. Mater. 2003, 15, 408-411.

(20) Gupta, S.; Zhang, Q.; Emrick, T.; Russell, T. P. Nano Lett. 2006, 6, 2066-2069.

(21) Ryan, K. M.; Mastroianni, A.; Stancil, K. A.; Liu, H.; Alivisatos, A. P. Nano Lett. 2006, 6, 1479-1482.

(22) Li, L., S.; Walda, J.; Manna, L.; Alivisatos, A. P. Nano Lett. 2002, 2, 557-560.

(23) Singh, A.; Gunning, R. D.; Ahmed, S.; Barrett, C. A.; English, N. J.; Garate, J.-A.; Ryan, K. M. J. Mater. Chem. 2012, 22, 1562-1569.

(24) Baranov, D.; Fiore, A.; van Huis, M.; Giannini, C.; Falqui, A.; Lafont, U.; Zandbergen, H.; Zanella, M.; Cingolani, R.; Manna, L. Nano Lett. 2010, 10, 743-9.

(25) Carbone, L.; Nobile, C.; De Giorgi, M.; Sala, F. D.; Morello, G.; Pompa, P.; Hytch, M.; Snoeck, E.; Fiore, A.; Franchini, I. R.; Nadasan, M.; Silvestre, A. F.; Chiodo, L.; Kudera, S.; Cingolani, R.; Krahne, R.; Manna, L. Nano Lett. 2007, 7, 2942-2950.

(26) Hung, A. M.; Konopliv, N. A.; Cha, J. N. Langmuir 2011, 27, $12322-12328$.

(27) Ahmed, S.; Ryan, K. M. Nano Lett. 2007, 7, 2480-2485.

(28) Ahmed, S.; Ryan, K. M. Chem. Commun. 2009, 6421-6423.

(29) Hung, A. M.; Oh, T.; Cha, J. N. Nanoscale 2012, 4, 1016-1020.

(30) Zanella, M.; Gomes, R.; Povia, M.; Giannini, C.; Zhang, Y.;

Riskin, A.; Van Bael, M.; Hens, Z.; Manna, L. Adv. Mater. 2011, 23, 2205-2209.

(31) Singh, A.; Gunning, R. D.; Sanyal, A.; Ryan, K. M. Chem. Commun. 2010, 46, 7193-7195.

(32) Rivest, J. B.; Swisher, S. L.; Fong, L., K.; Zheng, H.; Alivisatos, A. P. ACS Nano 2011, 5, 3811-3816.

(33) Singh, A.; Dickinson, C.; Ryan, K. M. ACS Nano 2012, 6, 33393345.

(34) Kang, C., C.; Lai, C., W.; Peng, H., C.; Shyue, J.-J.; Chou, P., T. ACS Nano 2008, 2, 750-756.

(35) Baker, J. L.; Widmer-Cooper, A.; Toney, M. F.; Geissler, P. L.; Alivisatos, A. P. Nano Lett. 2009, 10, 195-201.

(36) Renaud, G.; Lazzari, R.; Leroy, F. Surf. Sci. Rep. 2009, 64, 255380.

(37) Renaud, G.; Lazzari, R.; Revenant, C.; Barbier, A.; Noblet, M.; Ulrich, O; Leroy, F.; Jupille, J.; Borensztein, Y.; Henry, C. R.; Deville, J., P.; Scheurer, F.; Mane-Mane, J.; Fruchart, O. Science 2003, 300, 1416-1419.

(38) Bian, K.; Choi, J. J.; Kaushik, A.; Clancy, P.; Smilgies, D., M.; Hanrath, T. ACS Nano 2011, 5, 2815-2823.

(39) Hanrath, T.; Choi, J. J.; Smilgies, D., M. ACS Nano 2009, 3, 2975-2988.

(40) Disch, S.; Wetterskog, E.; Hermann, R. P.; Salazar-Alvarez, G.; Busch, P.; Brückel, T.; Bergström, L.; Kamali, S. Nano Lett. 2011, 11, $1651-1656$.

(41) Modestino, M. A.; Chan, E. R.; Hexemer, A.; Urban, J. J.; Segalman, R. A. Macromolecules 2011, 44, 7364-7371.

(42) Briki, F.; Busson, B.; Doucet, J. Biochim. Biophys. Acta 1998, 1429, 57-68.

(43) Denkov, N. D.; Velev, O. D.; Kralchevsky, P. A.; Ivanov, I. B.; Yoshimura, H.; Nagayama, K. Nature 1993, 361, 26.

(44) Phan, S. E.; Russel, W. B.; Zhu, J.; Chaikin, P. M. J. Chem. Phys. 1998, 108 (23), 9789-9795. 